\title{
Chance-constrained optimization of demand response to price signals
}

\author{
Dorini, Gianluca Fabio ; Pinson, Pierre; Madsen, Henrik
}

Published in:

IEEE Transactions on Smart Grid

Link to article, DOI:

10.1109/TSG.2013.2258412

Publication date:

2013

Link back to DTU Orbit

Citation (APA):

Dorini, G. F., Pinson, P., \& Madsen, H. (2013). Chance-constrained optimization of demand response to price signals. IEEE Transactions on Smart Grid, 4(4), 2072-2080. https://doi.org/10.1109/TSG.2013.2258412

\section{General rights}

Copyright and moral rights for the publications made accessible in the public portal are retained by the authors and/or other copyright owners and it is a condition of accessing publications that users recognise and abide by the legal requirements associated with these rights.

- Users may download and print one copy of any publication from the public portal for the purpose of private study or research.

- You may not further distribute the material or use it for any profit-making activity or commercial gain

- You may freely distribute the URL identifying the publication in the public portal

If you believe that this document breaches copyright please contact us providing details, and we will remove access to the work immediately and investigate your claim. 


\title{
Chance-constrained optimization of demand response to price signals
}

\author{
Gianluca Dorini, Pierre Pinson, Senior Member, IEEE, Henrik Madsen
}

\begin{abstract}
Household-based demand response is expected to play an increasing role in supporting the large scale integration of renewable energy generation in existing power systems and electricity markets. While the direct control of the consumption level of households is envisaged as a possibility, a credible alternative is that of indirect control based on price signals to be sent to these end-consumers. A methodology is described here allowing to estimate in advance the potential response of flexible end-consumers to price variations, subsequently embedded in an optimal price-signal generator. In contrast to some real-time pricing proposals in the literature, here prices are estimated and broadcast once a day for the following one, for households to optimally schedule their consumption. The price-response is modeled using stochastic Finite Impulse Response (FIR) models. Parameters are estimated within a Recursive Least Squares (RLS) framework using data measurable at the grid level, in an adaptive fashion. Optimal price signals are generated by embedding the FIR models within a chance-constrained optimization framework. The objective is to keep the price signal as unchanged as possible from the reference market price, whilst keeping consumption below a pre-defined acceptable interval.
\end{abstract}

Index Terms-demand forecasting, demand response, price signals, chance constrained optimization.

\section{INTRODUCTION}

I NTEGRATION of renewable though fluctuating energy generation, such as from wind and solar installations, is becoming an essential part of the development of future power systems and electricity markets. Relying on such energy sources with high variability and limited predictability propagates risk and uncertainty to the whole electricity value chain, challenging existing market structures and balancing strategies. The parallel phenomenon of household-type of end consumers becoming prosumers, both producing locally and envisaging a more proactive usage of electricity, challenges our traditional top-down approach to power system management. It will similarly affect the way electricity markets are designed and operated [1]. Such challenges in turn create opportunities, in the sense that demand-side management is foreseen to play a crucial role in providing the flexibility needed for load balancing and congestion control in systems with a high penetration of renewable energy generation. A representative set of recent work in that direction can be found in [2]-[7].

Various entities in power systems and electricity markets may be interested in optimally utilizing the flexibility of household-type of electricity consumers. In contrast to the direct control of households' consumption, where two-way communication is required so that the system operator may directly

The authors are with the Department of Applied Mathematics and Computer Science at the Technical University of Denmark (emails: gido@dtu.dk, ppin@dtu.dk,hmad@dtu.dk) define reference signals based on consumption feedback [8], the indirect control alternative using one-way communication only and based on price signals to be sent to households, is gaining increased interest. Concepts related to control in power systems based on prices have been discussed and studied for more than thirty years, as in [9]-[12] among others. It has been observed that flexibility is partly controllable by price variations as consumers become significantly priceelastic when exposed to varying prices [13], [14]. This requires that the price is efficiently displayed to final consumers [15]. Note that various views and definition of indirect control exist, as underlined in [16], while some potential limitations of dynamic price signals are also highlighted [17]. Two example applications of indirect control by price signals may be that of (i) a retailer aiming at revenue maximization by optimally trading its flexibility [18], and (ii) a demand-side aggregator wanting its pool of consumers targeting a reference in order to provide services to the grid [19]. Indirect control based on price signals has the advantage of neither requiring a bidirectional communication interface, nor knowledge of the end-user's environment.

The work in the present paper places itself in a framework different for other proposals in the literature, for a number of reasons. First of all, it is considered that price signals are optimized and broadcast once a day for the following one, for household-type consumers to optimally plan their consumption. They are therefore not generated in a model predictive control framework, as in [19] for instance. In parallel, the main objective when determining price signals is neither to minimize imbalances by explicitly shifting consumption in time, nor it is to attempt at having consumption following a certain reference signal. It is instead to ensure that consumption will stay as much as possible below a predefined acceptable level, for instance imposed by technical constraints at the grid level, or simply owing to market costs considerations for the aggregator/retailer providing electricity to these households. The importance of respecting grid-related operational constraints in demand-side management was recently exemplified for the case of electric vehicles in lowvoltage networks [20].

With these objectives in mind, our proposal is to use a data-driven statistical approach to estimate and forecast the dynamics of the consumers' elasticity. This task is performed using data measurable at grid level, removing the need to install sensors and communication devices between each individual consumer and the price-generating entity. This proposal contrasts with recent studies, where price response is assumed to be deterministic, also not being based on real data, e.g. [21], [22]. Here price signals are subsequently generated by em- 
bedding the forecasts (and their uncertainty) within a Chance Constrained (CC) optimization framework. The advantage of the proposed CC optimization approach is to explicitly account for uncertainty in the price response of consumers, as well as for a pre-defined maximum for allowed consumption levels, within the problem definition.

The paper is structured as following. In the first stage, Section II describes the mathematical background for the price-response model. This is followed in Section III by the presentation of the price signal generator in a chanceconstrained optimization framework. The application of that methodology to a real-world test is described in Section IV, based on a dataset composed by more than 500 households in Denmark subject to different price and control schemes. It is there shown how our proposal allows smoothing and moving consumption peaks. Concluding remarks end the paper in Section V.

\section{PRICE-RESPONSE MODEL}

The mathematical background of the price-response model follows that in [19], which extensively described a number of models for the dynamics of demand response to price signals. First, electricity consumption ought to be broken down into two additive components, that is, its non-responsive and responsive parts,

$$
c_{t}=f\left(\tilde{c}_{t-1}, \tilde{\mathbf{z}}_{t}\right)+g\left(\tilde{p}_{t}, \tilde{\mathbf{z}}_{t}\right),
$$

with

$$
\begin{aligned}
\tilde{c}_{t-1} & =\left[c_{t-1}, \ldots, c_{t-n_{c}}\right]^{\top} \\
\tilde{p}_{t} & =\left[p_{t+S}, \ldots, p_{t-L+S}\right]^{\top} \\
\tilde{\mathbf{z}}_{t} & =\left[\mathbf{z}_{t}, \ldots, \mathbf{z}_{t-n_{z}}\right]^{\top},
\end{aligned}
$$

where $n_{c}$ and $n_{z}$ denote the finite number of lags for past values of consumption $c$ and external variables $\mathbf{z}$ influencing consumption at time $t$. The price responsive component of the end-user consumption $\tilde{p}_{t}$ depends on a time window of $L$ price values, some before and some after the target time $t$. The number of future prices influencing the consumption is specified by the term $S \geq 0$. Following intuition, only the responsive part of the consumption is expected to be controllable with price signals.

Even though consumption is split conceptually, it is still the overall consumption that is to be modeled and predicted, with focus on the impact of price variations. In this paper the generic consumption model (1) is specified in the form of a Finite Impulse Response (FIR), see e.g. [23]. In such a form, price and external variables (outdoor temperature for instance) are decoupled, and the price response consequently isolated. A FIR model for $c_{t}$ can be expressed as a general linear model

$$
c_{t}=\tilde{c}_{t}^{\top} \boldsymbol{\theta}_{c}+\tilde{p}_{t}^{\top} \boldsymbol{\theta}_{p}+\tilde{\mathbf{z}}_{t}^{\top} \boldsymbol{\theta}_{z}+\epsilon_{t}=\mathbf{x}_{t}^{\top} \boldsymbol{\theta}+\epsilon_{t},
$$

where $\epsilon_{t}$ is a Gaussian noise with zero mean and finite variance, whereas $\boldsymbol{\theta}$ and $\mathbf{x}_{t}$ are defined as

$$
\begin{aligned}
\mathbf{x}_{t} & =\left[\tilde{c}_{t-1}^{\top}, \tilde{p}_{t}^{\top}, \tilde{\mathbf{z}}_{t}^{\top}\right]^{\top} \\
\boldsymbol{\theta} & =\left[\boldsymbol{\theta}_{c}^{\top}, \boldsymbol{\theta}_{p}^{\top}, \boldsymbol{\theta}_{z}^{\top}\right]^{\top} .
\end{aligned}
$$

The vector of coefficients $\boldsymbol{\theta}_{p}$ corresponding to the price input variable defines the impulse response function from price to consumption, characterizing the price-response in $g$.

At time $t$, the optimal $k$-step consumption prediction minimizing squared errors is the conditional expectation [23]. For the FIR model (2), this forecasting is given by

$$
\hat{c}_{t+k \mid t}=\mathbb{E}\left\{c_{t+k} \mid \hat{\mathbf{x}}_{t+k \mid t}\right\}=\hat{\mathbf{x}}_{t+k \mid t}^{\top} \boldsymbol{\theta} .
$$

The input vector $\hat{\mathbf{x}}_{t+k \mid t}$ of explanatory variables is noted as a forecast since it may indeed include predictions of certain variables, e.g., price and temperature at time $t+k$.

In order to estimate the coefficients in the FIR model (2), recursive and adaptive estimation is used based on Recursive Least Squares (RLS). For an introduction to RLS estimation in FIR models, the reader is referred to Ref. [23]. It consists in updating the model coefficients at every time $t$ when new data becomes available, with the following two-step procedure,

$$
\begin{aligned}
\mathbf{R}_{t} & =\alpha \mathbf{R}_{t-1}+\mathbf{x}_{t} \mathbf{x}_{t}^{\top} \\
\hat{\boldsymbol{\theta}}_{t} & =\hat{\boldsymbol{\theta}}_{t-1}+\mathbf{R}_{t}^{-1} \mathbf{x}_{t}\left(y_{t}-\mathbf{x}_{t}^{\top} \hat{\boldsymbol{\theta}}_{t-1}\right) .
\end{aligned}
$$

In order to avoid computational issues related to matrix inversion, $\mathbf{R}_{t}$ should be initialized with sufficiently small values and not inverted before, say, 100 matrix updates. Similarly, the various explanatory variables whose successive values compose $\mathbf{x}$ should be normalized. The vector $\hat{\boldsymbol{\theta}}_{t}$ of model coefficients can be initialized with a vector of zeros.

Here it is assumed that the dependency between consumption, price, and the other external variables, can be described using the general linear model (2). If for some other test cases this assumption was not deemed acceptable, nonlinearities could be included in different ways. For instance by using basis functions in a linear approximation, e.g., with polynomial and spline bases, or Fourier and exponential series. A discussion of methods permitting to handle the nonlinear case, including recursive and adaptive estimation, can be found in Ref. [19].

\section{Generation of Optimal Price Signals}

The price-response model discussed in Section II allows predicting and simulating electricity consumption under multiple pricing scenarios, along with its uncertainty. It can for instance be used by a Balance Responsible Party (BRP) as a tool to evaluate and compare the effects of different price variations. Formally, a BRP has the responsibility to insure the match between supply and consumption of electric energy in its balance area, while being financially penalized for any deviation that may arise. Effectively here, the price-response model can be embedded in a price signal optimization strategy, whose purpose is to optimize prices in view of the BRP's objectives, as well as of the potential flexibility of consumers.

At every time $t$ a sequence of $K$ future price scenarios $p_{t+k}, k=1, \ldots, K$, can be generated, and the corresponding consumption response $c_{t+k \mid t}, k=1, \ldots, K$, simulated with Eq. (2). The consumption values based on the reference market prices $\bar{p}_{t+k}, k=1, \ldots, K$, are denoted by $\bar{c}_{t+k \mid t}, k=$ $1, \ldots, K$. Now suppose that this reference consumption scenario happens to exceed a pre-defined sequence of maximum 
consumption levels $c_{t+k}^{\max }, k=1, \ldots, K$, where $c_{t+k}^{\max }$ may for instance originates from grid constraints at the distribution level (as for the EV charging problem of [20]) or decided upon owing to expected prohibitive balancing costs. The BRP would then like to incentivize modifications in consumption so as to stay as much as possible below that maximum level, by introducing deviations in prices from its market values. This is done here by setting up anoptimization problem imposing a level of confidence $1-\rho$ on the maximum allowed consumption level,

$$
\mathrm{P}\left[c_{t+k \mid t}>c_{t+k}^{\max }\right]<\rho, \quad k=1, \ldots, K
$$

where $c_{t+k \mid t}, k=1, \ldots, K$ is the modified consumption, induced by changes in prices. Note that there is still a probability $\rho$ of exceeding the constraint $c_{t+k}^{\max }$.

Let $\mathcal{F}_{t}$ be the information set available at time $t$, i.e., containing the measurements and potentially forecasts of explanatory variables needed as input to the FIR model (2). The optimization objective to be minimized is defined as the deviation between the potential temporal evolution of consumption (which we refer to as trajectory or scenario) over the $K$ lead times if influenced by price signals, and the consumption trajectory that would realize if no price signal was used,

$$
\min _{p_{t+1}, \ldots, p_{t+K}} \mathbb{E}\left[\sum_{k=1}^{K}\left(c_{t+k \mid t}-\bar{c}_{t+k \mid t}\right)^{2} \mid \mathcal{F}_{t}\right] .
$$

By plugging the linear form of (2) into (6), we obtain a quadratic objective function to be minimized,

$$
\min _{p_{t+1}, \ldots, p_{t+K}} \sum_{k=1}^{K}\left(\tilde{p}_{t+k}^{\top} \boldsymbol{\theta}_{p}-\tilde{\bar{p}}_{t+k}^{\top} \boldsymbol{\theta}_{p}\right)^{2},
$$

also being a direct function of the price signals $p_{t+1}, \ldots, p_{t+K}$, which are the decision variables of the optimization problem.

Prices are also assumed to be non-negative, hence a further constraint is imposed

$$
p_{t+k} \geq 0, k=1, \ldots, K .
$$

The optimal solution is the price signal $p_{t+k}^{*}, k=1, \ldots, K$ and corresponding consumption $c_{t+k}^{*}, k=1, \ldots, K$, attaining the minimum of (7) whilst fulfilling the constraint in (5).

As a result of the above optimization problem, prices are issued so that operational constraints are fulfilled with a minimum impact over the reference household consumption, namely the consumption that would have occurred if disregarding operational constraints. Note that the potential prices variations are here neither bounded nor directly penalized, though they could be, for instance in line with the recent proposal in Ref. [18] where the allowed range for price variations is seen as defined through retailer-consumer contracts or through regulation. Such aspects could be accounted for in the above optimization problem by adding a penalization of prices and their variations in the objective function, or by introducing additional constraints reflecting an agreed price range. It is also important to notice how the proposed CC approach does not attempt to impose a specific time pattern to the end-users consumption, as would be the case with model predictive controllers e.g. [19]. In a way this approach is more flexible, as it allows for any consumption pattern, provided that the probability to exceed constraint (5) is lower than the desired probability $\rho$.

This type of CC optimization formulation has been extensively studied in Operations Research. Applications of CC optimization (or CC programming), are rather broad: reservoir operation [24], ground-flow management [25], portfolio management [26], chemical engineering [27], and power system studies [28]-[30], among others. For extensive reviews on the theory and applications of CC optimization problems, the reader is referred to [31], [32]. Here we limit the discussion to showing how, under the assumption of linearity and Gaussianity, the $\mathrm{CC}$ is equivalent to a deterministic convex programming problem, and to how it can thus be solved efficiently.

Since the price-response model (2) is a linear one, assuming that the random variables $\epsilon_{t}$ are Gaussian and independent, the objective function (7) has a quadratic form, and the constraints (5) can be expressed based on quantiles with nominal proportion $1-\rho$ for the predictive distribution of consumption,

$$
\tilde{c}_{t+k}^{\top} \boldsymbol{\theta}_{c}+\tilde{p}_{t+k}^{\top} \boldsymbol{\theta}_{p}+\tilde{\mathbf{z}}_{t+k}^{\top} \boldsymbol{\theta}_{z}+\sqrt{\operatorname{Var}\left\{\epsilon_{t+k}\right\} q_{1-\rho}^{N(0,1)}}<\rho
$$

where $q_{1-\rho}^{N(0,1)}$ is the quantile with nominal proportion $1-\rho$ of a standard Gaussian variable $N(0,1)$. The quantile in (9) enters a linear inequality directly influenced by the decision variables. The resulting CC problem formulation has quadratic objective function and linear inequality constraints. It can be written in a compact manner as

$$
\begin{array}{ll}
\min _{p_{t+1}, \ldots, p_{t+K}} & \sum_{k=1}^{K}\left(\tilde{p}_{t+k}^{\top} \boldsymbol{\theta}_{p}-\tilde{\bar{p}}_{t+k}^{\top} \boldsymbol{\theta}_{p}\right)^{2} \\
\text { s.t. } & \tilde{c}_{t+k}^{\top} \boldsymbol{\theta}_{c}+\tilde{p}_{t+k}^{\top} \boldsymbol{\theta}_{p}+\tilde{\mathbf{z}}_{t+k}^{\top} \boldsymbol{\theta}_{z} \\
& +\sqrt{\operatorname{Var}\left\{\epsilon_{t+k}\right\} q_{1-\rho}^{N(0,1)}}<\rho, k=1, \ldots, K \\
& p_{t+k} \geq 0, k=1, \ldots, K
\end{array}
$$

where the $p_{t+k}$ 's are the $K$ decision variables, and where $\rho$ is an input parameter defining the probability that the operational constraint is not respected.

The reader can easily verify the convexity of the quadratic form of (6), hence yielding a convex optimization problem. The optimal solution can be obtained by a straightforward application of Interior Point (IP) methods, already extensively employed for a number of practical applications [33], and available as part of off-the-shelf optimization solvers.

In general an IP method is an iterative procedure, where each step requires the calculation of the first and second order derivatives of the objective functions and constraint functions. For many practical applications interior-point methods can solve the problem in a number of steps or iterations that is almost always in the range between 10 and 100. Ignoring any structural peculiarity of the problem (such as sparsity), each step requires on the order of $\max \left\{n^{3}, n^{2} m, F\right\}$ operations, where $n$ is the number of decision variables, $m$ is the number 
of constraints, and $F$ is the cost of evaluating the derivatives. Description of IP methods and descent methods, as well as further analysis of their complexity, can be found in [34].

\section{APPLICATION TO PRICE-RESPONSIVE HEATING SYSTEMS}

A case study is presented in the following, showing how, using the above methodological proposal, household heating can be used to bring flexibility into the power system. Household heating systems can be seen as flexible devices, hence endowed with inertia due to the time constants involved in their heat dynamics. Other flexible devices that could be considered include cooling systems, hot tap water heating, space heating, air conditioning systems, etc. Flexible devices have the property such that they can be turned off (or more generally, pushed away from their reference functioning point) during a short period of time with no or very little consequence on the comfort of household occupants. Such feature can be exploited to provide services to the power system in a market environment.

The experimental data was collected in the frame of the DEVI experiment, within the FlexPower project [14], as first described below. Subsequently, the way the FIR models were fitted is presented, followed by a visualization of the consumption response to prices identified from the experimental data. Finally, the chance-constrained optimization used for price generation is illustrated.

\section{A. The DEVI experiment}

The experiment was conducted in South Jutland (Denmark) to identify and measure the response from more than 500 households subject to different kinds of schemes for regulating their electricity consumption.

All households in this experiment have a high consumption of electricity for heating (more than $15000 \mathrm{kWh} /$ year). In the winter period, in fact, the electricity consumption is about 3.5 times more than in the summer period. Different regulation methods have been used:

- Electronic housekeeper (20 households) - An installed electronic system that shows the price signals, to which the users can manually respond to by turning the heating up and down;

- Email (114 households) - The users receive a daily mail containing the prices for the next day, inducing a potential manual response;

- SMS (35 households) - The users receive a daily SMS containing the prices for the next day, inducing a potential manual response;

- DEVI (16 households) - An installed system that collects the prices and automatically regulates the heating so that more electricity is used when prices are low and less is used when prices are high. The individual households have a certain degree of control over the equipment and can e.g. decide how high and low the inside temperature is allowed to be;

- Control group (355 households) - No specific installed system, while not receiving any price information.
All households in the experiment eventually received electricity priced as 'spot price with financing', which in practice is the market price plus an overhead of 1-2 $\varnothing$ re (hundreds of Danish Crown). Data was collected over a period between April 2007 and March 2009. The data points are hourly measurements of electricity consumption, but not for the individual households, rather as mean values for every group.
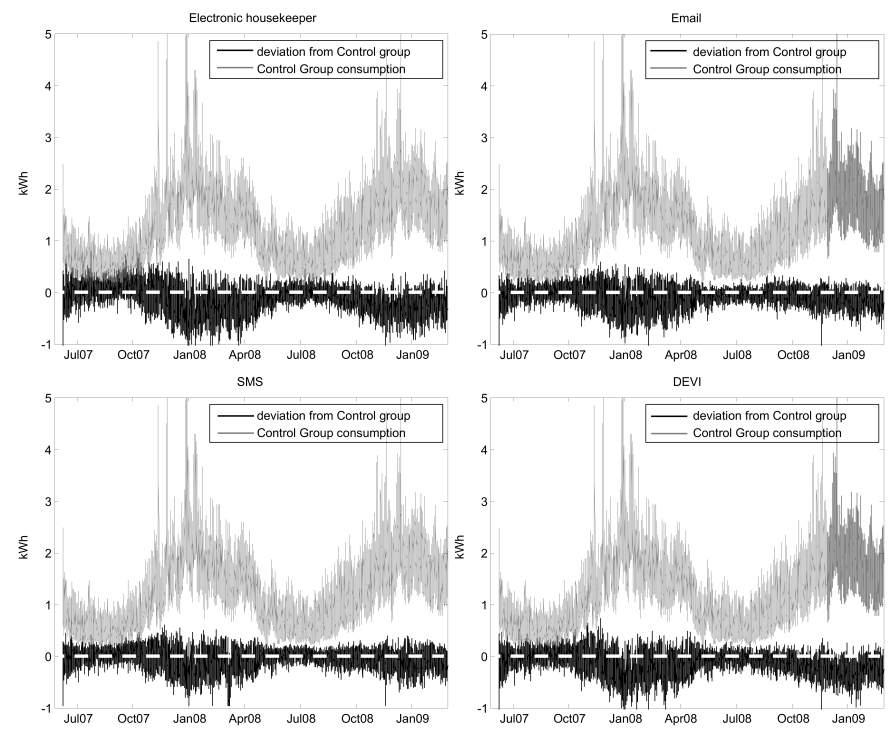

Fig. 1. Deviation in consumption between the control group and the priceresponsive groups.

The price-responsive behavior can be qualitatively observed by confronting the consumption of the differently regulated groups against that of the control group. Fig. 1 shows that consumption patterns are similar during the summer period. During the winter instead, when a significant overhead is brought in by heating costs, the consumption of the various regulated groups tends to be lower than for the control group.

\section{B. Estimation of the price response}

The generic model structure presented in Section II is here adapted to case of the DEVI dataset. In line with the experimental setup, consumption time series are considered at an aggregated level, not for individual households.

The control group serves as a reference to monitor the behavior of consumers not being responsive to price variations. The hypothesis is made that for all other groups, if they were not price-responsive, they would follow the same consumption pattern as for the control group. The approach therefore consists in modeling the control group consumption, and then the deviation of each price-responsive groups from this reference pattern. Furthermore, price responsiveness is assumed only for heating, as winter electricity consumption weights more than $70 \%$ of total annual consumption.

The data available, as envisaged in an indirect control framework, does not make distinction between heating and not heating (seen a base consumption) usage of electricity. This is somehow handled by the models based on the previously formulated assumption that it is the heating part of the consumption that is responsive to prices. 
The comfort needs of the electricity consumers vary during the day, mostly depending on whether or not they are at home, thus the base consumption is a time-dependent variable. Here a simple look-up table representation is adopted, where the time variable is classified in categories. We denote by $I$ the set of conditions, and with $b(i, t)$ a Boolean function returning 1 if condition $i \in I$ is true at time $t$, and zero otherwise. For each $t$ there is one and only one $i \in I$ such that $b(i, t)=1$. Hence $b(j, t)=0$ for all $j \neq i$.

The only external variable considered is a function of the external temperature, denoted by $T$, being the signed deviation of actual temperature from a reference of $17^{\circ} \mathrm{C}$. This deviation is calculated based on a weighted average of the actual temperature observations over the previous 72 hours. If the temperature is above $17^{\circ} \mathrm{C}$, then $T$ is set to $0 . T$ can then be seen as a variable directly inducing needs for electric heating.

The control group consumption $c^{C G}$ is described with the linear model

$$
\begin{aligned}
c_{t}^{C G} & =\sum_{i=1}^{N} \theta_{c, j} c_{t-i+1}^{C G}+ \\
& +\sum_{i=1}^{M} \theta_{T, i} T_{t-i+1}+\sum_{i \in I} \theta_{b, i} b(i, t)+\varepsilon_{t}^{C G},
\end{aligned}
$$

where $\varepsilon_{t}^{C G}$ is a centered Gaussian noise with finite variance $\sigma^{C G}$. The model complexity is chosen such that an increase in the number of lags does not significantly improve performance, measured by a coefficient of determination $R^{2}$.
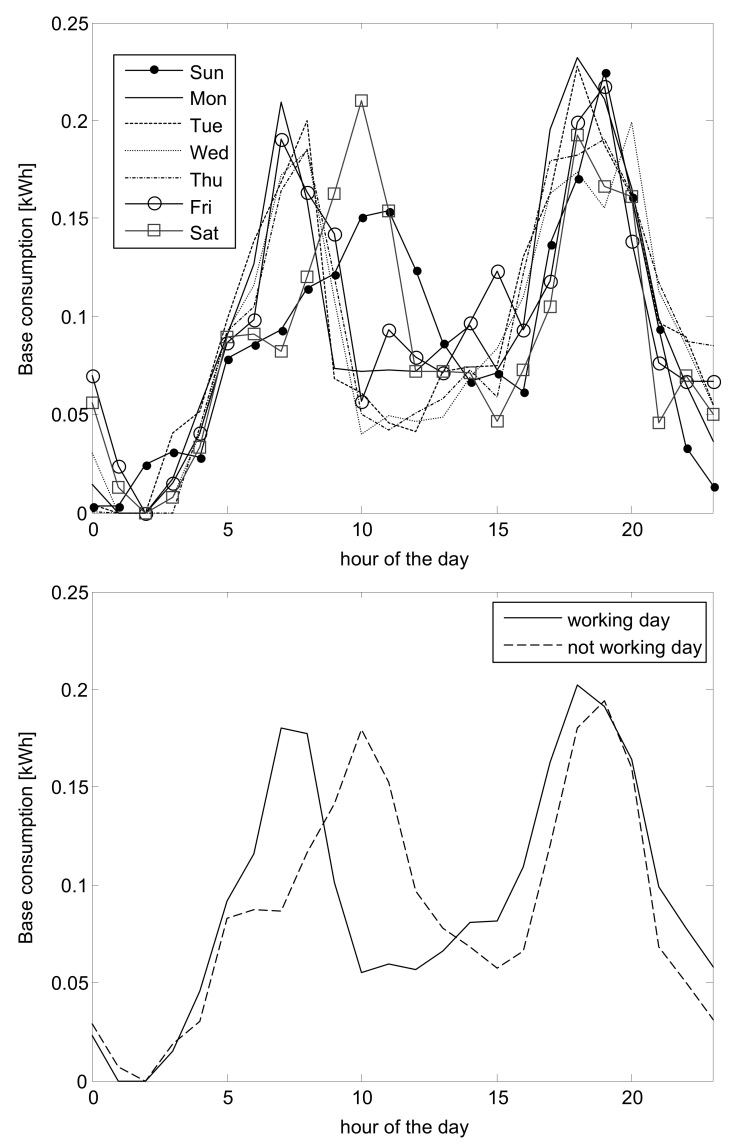

Fig. 2. Two equivalent categorization for the base consumption.
The choice of the categories $I$ is done by combining different types of classification, such as hour of the day, day of the week, month, season, working day, holiday, etc. It is important to keep in mind that the number of categories can grow very fast. Large number of categories increases model complexity, and affects its generalization ability. On the other hand, different classifications may be equivalently descriptive even though leading to significantly different number of parameters. For instance, Fig. 2 shows how the time of the day $\times$ working-day/holiday classification, having 14 categories only, can be used instead of time of the day $x$ day of the week, yielding 168 categories. Both representations, in fact, show that the daily base consumption has two peaks, one in the morning and one in the evening around 19:00. The peak in the morning is around 7:00 during the working days, and around 10:00 during the non working days.

The selected model has parameterization $N=3, M=1$, $|I|=14$, with a resulting coefficient of determination of $94 \%$. The reduced number of past temperature terms $M$ should not be surprising, as the variable $T$ is time aggregated and thus it represents a cumulative indicator of the energy needs for heating.

The model, whose parameters are estimated adaptively using a forgetting factor $\alpha=0.995$, allows for stable extended stochastic simulations, as shown in Fig. 3. Perhaps, higher performance could be obtained by selecting $\alpha$ with a proper cross-validation exercise, like illustrated in [23]. However, even with the actual parameterization, both the whiteness and the Gaussianity (Fig. 4) of the residual prediction error $\epsilon_{t}^{C G}$ could be confirmed.

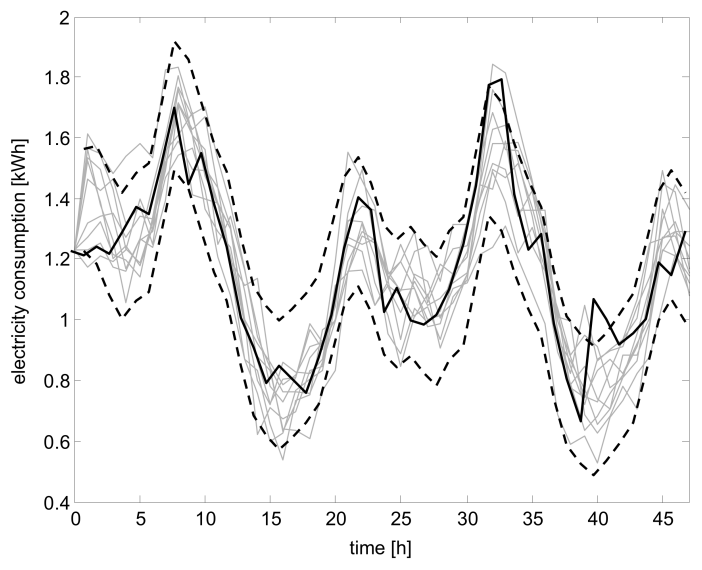

Fig. 3. Stochastic simulation of the control group consumption. Solid line are measured data, dashed lines are the $95 \%$ prediction interval, grey lines are consumption scenarios obtained by Monte Carlo simulation.

The response of consumers to prices for the $i$-th group is modeled as the deviation from the control group,

$$
\begin{aligned}
\left(c_{i, t}-c_{t}^{C G}\right) & =\sum_{j=1}^{R} \theta_{c, i, j}\left(c_{i, t-j}-c_{t-j}^{C G}\right)+ \\
& +\sum_{j=1}^{L} \theta_{p, i, j} p_{t-j+S}+\sum_{j=1}^{R_{e}} \theta_{e, i, j} e_{t-j}+\varepsilon_{t}
\end{aligned}
$$




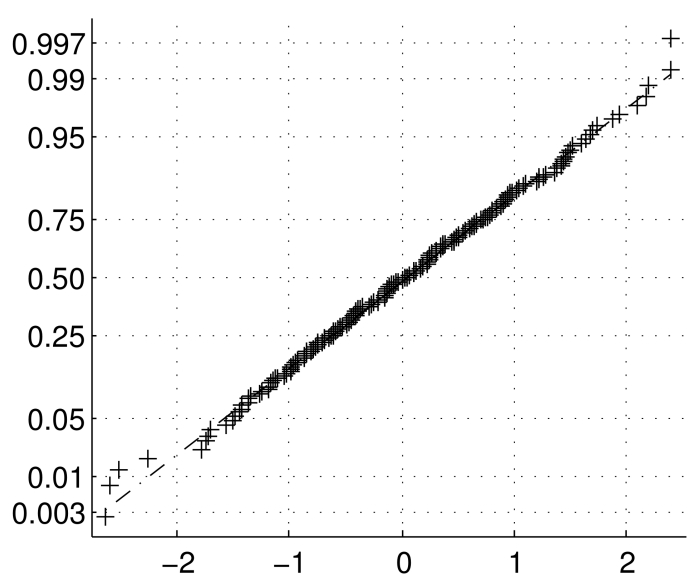

Fig. 4. Gaussian quantile-quantile plot for the control group model residuals $\epsilon_{t}^{C G}$ collected up to 4 Nov. 2008.

with $\varepsilon_{t}$ a centred Gaussian noise of finite variance, and where the terms $e_{t}$ are the past prediction errors, thus yielding a moving average component. The deviations in consumption with respect to the control group is based on a certain number $S$ of future prices.

The optimal parameterization of the selected models was obtained after maximization of the coefficient of determination $R^{2}$. The same parameterization was chosen for all models. The price response term covers a time window of $L=36$ hours, with a look ahead time of $S=24$ hours. The moving average counts $R_{e}=24$ past prediction errors, whereas only one autoregressive term $R=1$ was needed. Parameters were estimated adaptively using a forgetting factor $\alpha=0.995$, yielding a coefficient of determination of $77 \%$ for the electronic housekeeper group, $82 \%$ for the DEVI group, $86 \%$ for SMS and Email groups. Fig. 5 shows an example of scenarios obtained by Monte Carlo simulation, along with estimated prediction intervals, for the DEVI group. The Gaussian quantile-quantile plots of Fig. 6 illustrate that the model residuals are very close to being Gaussian, maybe except in the very tails for the DEVI group.

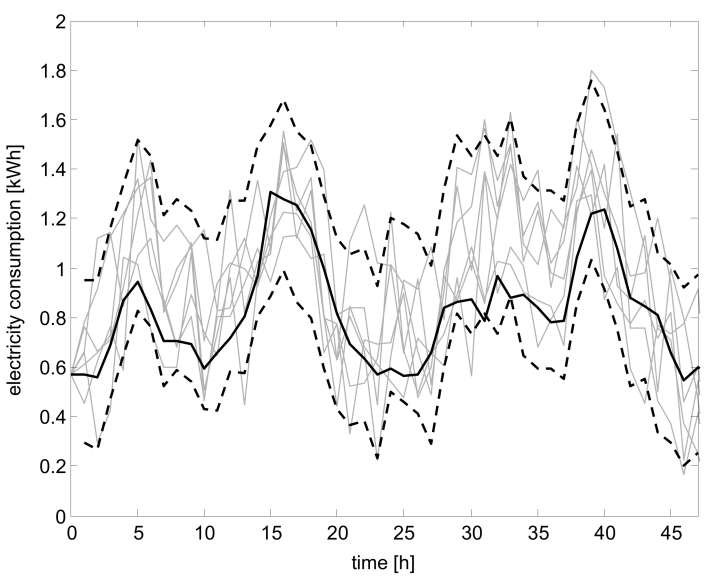

Fig. 5. Stochastic simulation of the DEVI group consumption. The solid line is for the measured data, dashed lines are for the $95 \%$ prediction interval, while the grey lines are the scenarios obtained by Monte Carlo simulation.

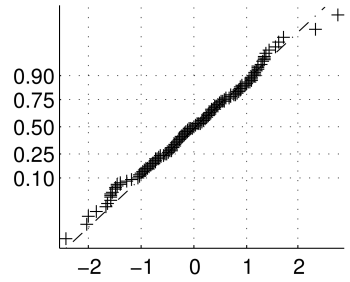

(a) Elect Housekeepers

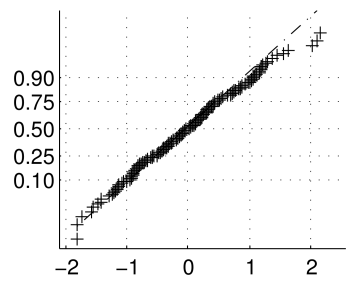

(c) SMS

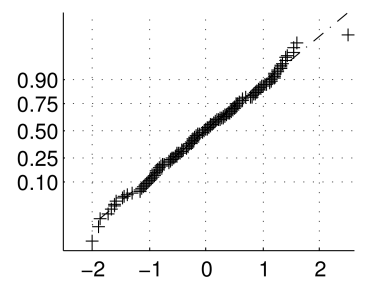

(b) Email

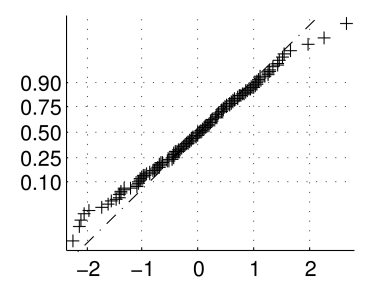

(d) DEVI
Fig. 6. Gaussian quantile-quantile plots for the model residuals $\epsilon_{t}^{i}$ for the various regulated groups, collected up to 4 Nov. 2008.

\section{Visualization of the price response}

The FIR models fitted previously may then be used to visualize the nature and dynamics of the response to price signals as identified based on the experimental data. In practice, this is done by isolating the change in electricity consumption induced by a unit step change in price-for instance, and increase of 1 Danish Crown at a given time $t$. For that purpose, we exploit the superposition principle, which is valid for linear systems [23]. It translates to calculating the difference in consumption between two simulations, one being the reference scenario with constant electricity prices, and the other one having the same characteristics except for a change of one unit in the price at a given time $t$. Both simulations have the same duration and initial conditions, while they ought to be long enough to ensure convergence so that the whole price response pattern is observed. In the present case, the results obtained for the various groups and their associated fitted FIR models are gathered in Fig. 7. In this figure, the step change in price occurs at the " 0 " time index.

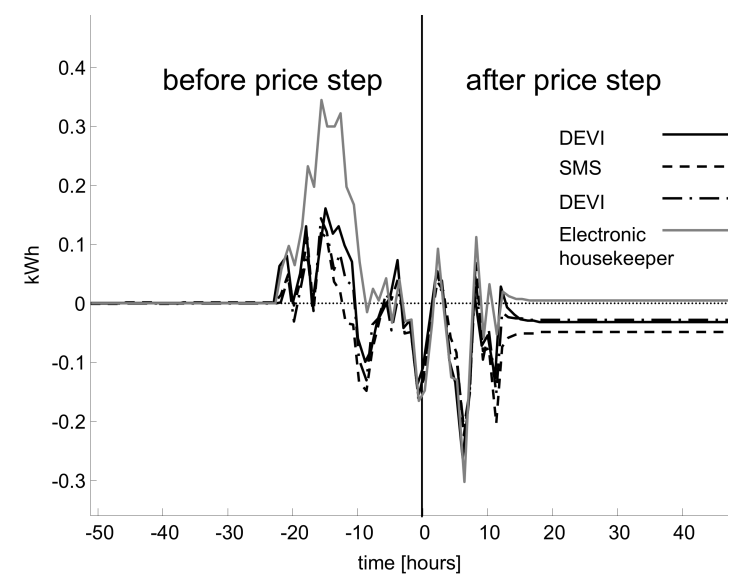

Fig. 7. Response of consumption to a step change of 1 Danish Crown in price, as described by the FIR models fitted to the experimental data. 
All groups respond by increasing the consumption before the price increment, and by decreasing the consumption after the price increment. As expected, pre-heating takes place during periods of low prices in anticipation for future higher prices. Normally, price fluctuations take place during a short period of time, whereas it takes a certain time for the household temperature to reach its reference. Therefore, inducing changes in household electricity consumption by sending price signals barely reduced their comfort. If the price remains high, the consumer groups reduce consumption, hence they reduce the temperature in the house and the corresponding comfort level. The only exception is for the electronic housekeeper group. The pre-heating in this case is significantly higher than the other groups, but eventually, if the price stays high, the consumption remain unchanged.

Besides the noise caused by the limited sample size of the regulated households (i.e., the number of participants in the regulated experiments), the rather harsh shape of the stepresponse function in Fig. 7 may be also interpreted as a consequence of very nature of the demand response mechanism. Indeed, electricity consumers are not bounded to any specific responsive mechanism (since manual), hence their individual response can be fairly diverse.

\section{Control by price using chance constrained optimization}

The FIR model for the DEVI dataset, described in Section IV-B, is here embedded within the $\mathrm{CC}$ approach for the determination of optimal price signals, in turn described in Section III. As an illustrative example, we consider a situation where the price signal is unique, and we test the $\mathrm{CC}$ optimization assuming that the price-response mechanism is represented by the DEVI group. In fact, among the four groups considered, DEVI is the only one equipped with fully automatic responding devices, hence reflecting the ideal population of flexible electricity consumers.

We address the situation where, at time $t$, a possible issue with consumption reaching a level above the capacity limit is foreseen over the range of the $K$ lead times. Such situation is depicted in Fig. 8, where the system capacity limit $c_{t, 1}^{\max }, k=$ $1, \ldots, K$ shows a drop. This passing of system capacity limits is assessed by the BRP, using the FIR model to simulate the end-user price response, based on the temperature forecast, and on market prices. Consequently, the BRP iterates on potential price signals through the CC optimization approach, then eventually sending an optimized prize signal back to the households.

The reference set of prices $\left\{p_{t, 1}, \ldots, p_{t, K}\right\}$ is optimally determined by imposing a level of confidence $1-\rho$ of not exceeding the pre-defined maximum allowed consumption for the group, as in (5). Since the DEVI model described in Section IV-B is linear, the system capacity constraints in (5) take the linear form of (9), while the objective function (7) in the CC optimization is quadratic. The optimal solution is found using IP methods and the optimized set of price signals $\mathbf{p}_{t}^{*}$ is sent to the households. For each value of $\rho$, the optimization took less than a tenth of a second, using a standard quadratic programming solver coded in MATLAB.
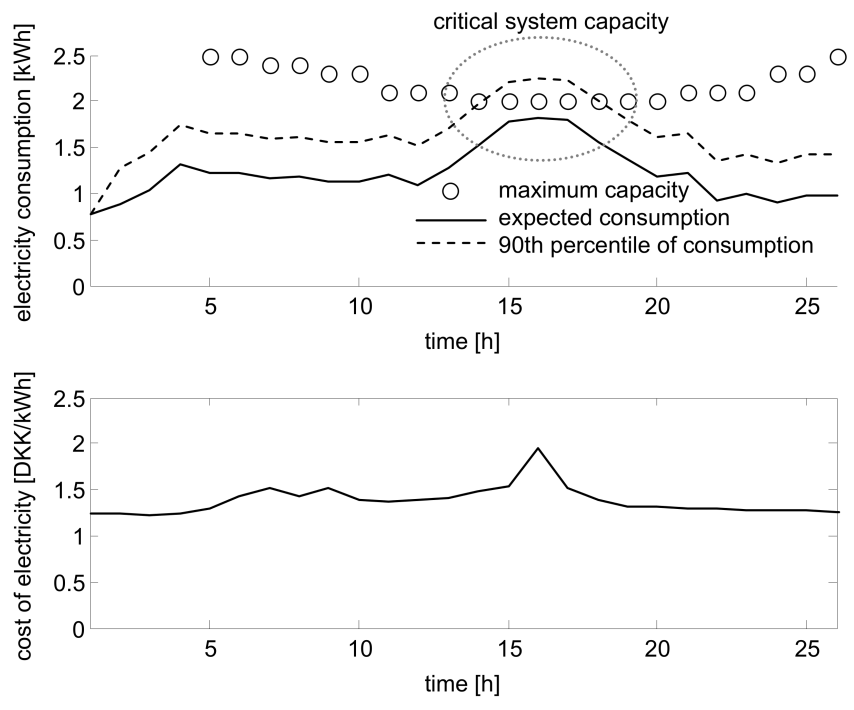

Fig. 8. Example of a scenario where pre-defined maximum consumption level $c_{t, k}^{\max }$ is likely to be exceeded by the participants in the DEVI group.

Market prices and electricity consumption are mutually dependent, as they form an equilibrium. This can be observed in the critical scenario of Fig. 8, where the price peak corresponds to a higher consumption level. The CC optimization framework is conceived in order to alter the priceconsumption equilibrium as little as possible. The impact of the optimization on the original price signal can be observed from Figs. 9, 10, and 11, where the CC problem is solved for increasing levels of confidence $1-\rho$. In all cases, the optimized price tends to anticipate the price peak, triggering a smoother and distributed consumption response. The impact of the optimization on the original price signal grows with the desired level of confidence $1-\rho$ assigned to the pre-defined maximum allowed consumption level.
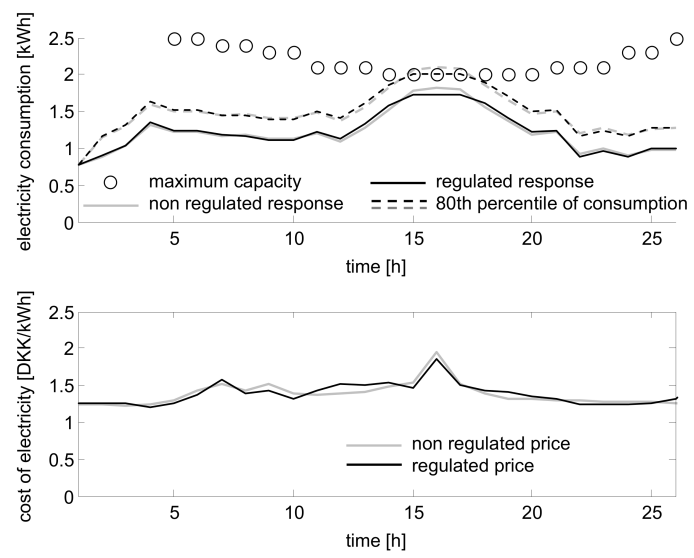

Fig. 9. DEVI group consumption response to $\mathrm{CC}$ optimized price signals, when the confidence level is $1-\rho=80 \%$.

It is important to notice how peaks and fluctuations in both the optimized price signal $\mathbf{p}_{t}^{*}$ and related successive consumption values $c_{t, k}^{*}, k=1, \ldots, K$ are similar to the reference scenario $\bar{p}_{t, k}, k=1, \ldots, K$, and $\bar{c}_{t, k}, k=1, \ldots, K$. More specifically, when $1-\rho=95 \%$ (the most constraining case), there is a maximum deviation of $41 \%$ in price, and 

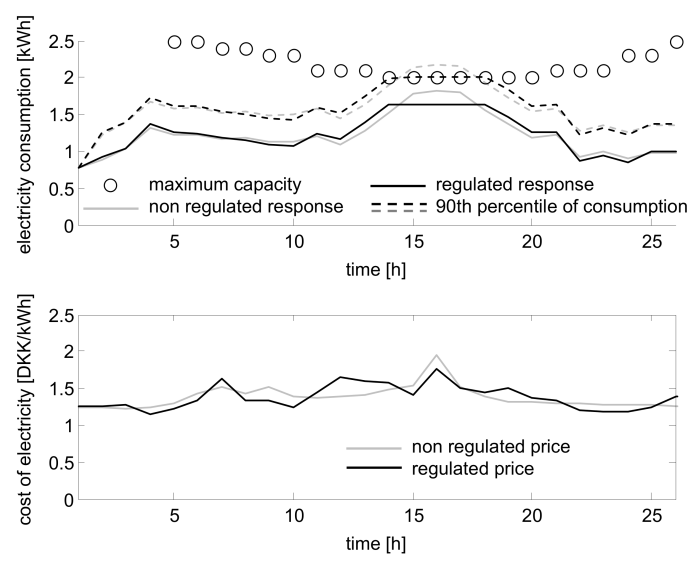

Fig. 10. DEVI group consumption response to $\mathrm{CC}$ optimized price signals, when the level of confidence is $1-\rho=90 \%$.
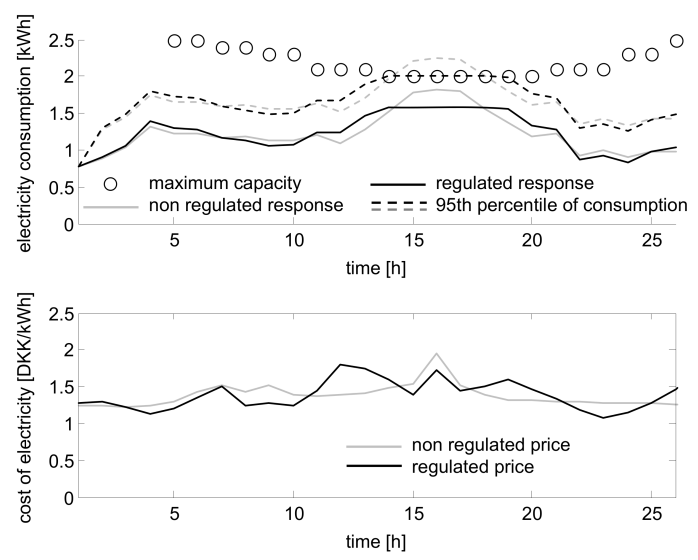

Fig. 11. DEVI group consumption response to CC optimized price signals, when the level of confidence is $1-\rho=95 \%$.

$16.5 \%$ in consumption. Meanwhile, this translate to an increase of total costs for the consumer of only $1.2 \%$ with an overall consumption decrease of $0.22 \%$. This suggests that, both in terms of total costs and consumption, the price signal has significant short-term impact, and close to negligible impact on overall consumption and costs for the consumers. It also shows that realistic price signals can be generated even without imposing tight price constraints, and without attempting to force the consumption through a specific time pattern. In the presented CC formulation, in fact, the only price constraint is non-negativity (Eq. (8)), whereas the consumption can have any pattern not exceeding the pre-defined maximum constraint.

\section{COnCluding Remarks}

The price-responsive consumption of household-type of electricity consumers was modeled using stochastic FIR models, hence also accounting for uncertainties in their consumption pattern and response to price variations. Models parameters were adaptively estimated in a RLS framework, permitting to track smooth changes in consumer's response to price variations, which may naturally change with time and seasons in the case of electric heating. Recursivity additionally allows decreasing computational costs - a nice feature when aiming to embed predictions and scenarios in a realworld optimization problem. Even though FIR models were deemed sufficiently accurate for describing the dynamics of consumption as induced by external weather variables and price variations, it may be that more advanced modelling approaches could be used in the future, potentially allowing for more accurate and skillful forecasts of consumption dynamics. This would come at a cost though, since embedding nonlinear models and resulting forecasts in a price generator would most certainly result in more complex optimization problems.

The price signal was optimized by embedding the FIR model within a $\mathrm{CC}$ optimization framework. The $\mathrm{CC}$ optimization problem may be defined by the BRP, setting a time sequence of maximum admissible loads over a set of lead times. Those maximum acceptable values may be motivated by technical constraints at the grid level, or simply owing to market costs considerations for the aggregator/retailer providing electricity to these households. The BRP can set the level of confidence for the maximum consumption limits, and the CC optimization yields an optimal price signal fulfilling the constraints with a minimum deviation from the original consumption pattern. The optimization criterion is based on the consideration that market prices and consumption levels are mutually dependent. The linearity of the price-response models is exploited so that the CC problem is quadratic and convex. Optimal price signals can then be readily obtained using IP methods.

The methodology was applied to the real-world test case of the DEVI experiment in order to show its practical applicability. The way peaks in consumption may be smoothed in time by anticipating price peaks was illustrated. Following intuition, the higher the level of confidence for maximum load constraints is, the more the optimized price deviated from the market price.

This proposal methodology comprises an alternative to existing proposals in a model predictive control framework, hence considering real-time dynamic pricing. Optimal determination and broadcast of prices a fair amount of time in advance permits to plan consumption in a way that may not be possible with real-time pricing. In practice, it is believed that the two approaches ought to be combined: similarly to current market organization today, demand-side management based on price signals should consist of two stages, i.e., the day-ahead optimization of consumption patterns in view of dynamic operational constraints, and a real-time balancing stage based on real-time pricing. The respective advantages and drawbacks of these complementary approaches, both in terms of economics and power system aspects, should be the focus of future work.

\section{ACKNOWLEDGMENT}

The work presented was partly supported by the Danish Public Service Obligation (PSO) Fund, under the FlexPower project (contract no. 2010-1-0486), as well as by the iPower platform project, supported by DSF (Det Strategiske Forskningsråd) and RTI (Rådet for Teknologi og Innovation), which are hereby acknowledged. The authors additionally acknowledge the four reviewers and the editor for their comments and suggestions. 


\section{REFERENCES}

[1] R. Schleicher-Tappeser, "How renewables will change electricity markets in the next five years," Energ. Policy, vol. 48, pp. 64-75, 2012.

[2] J. Torriti, M. Hassan, and M. Leach, "Demand response experience in Europe: Policies, programmes and implementation," Energy, vol. 35, no. 4, pp. $1575-1583,2010$.

[3] G. Strbac, "Demand side management: Benefits and challenges," Energ. Policy, vol. 36, no. 12, pp. 4419 - 4426, 2008.

[4] P. Nyeng and J. Østergaard, "Information and communications systems for control-by-price of distributed energy resources and flexible demand," IEEE Trans. Smart Grid, vol. 2, no. 2, pp. 334-341, 2011.

[5] A. Mohsenian-Rad, "Optimal residential load control with price prediction in real-time electricity pricing environments," IEEE Trans. Smart Grid, vol. 1, no. 2, pp. $120-133,2010$.

[6] A. Conejo, J. Morales, and L. Baringo, "Real-time demand response model," IEEE Trans. Smart Grid, vol. 1, no. 3, pp. 236 - 242, 2010.

[7] A. Keane, A. Tuohy, P. Meibom, E. Denny, D. Flynn, and A. Mullane, "Demand side resource operation on the irish power system with high wind power penetration," Energ. Policy, vol. 39, no. 5, pp. 2925 - 2934, 2011.

[8] B. Biegel, "Flexibility interface - Information modeling for direct control," iPower project, Tech. Rep., 2012, available at: ipower-net.dk.

[9] F. Schweppe, R. Tabors, J. Kirtley, H. Outhred, F. Pickel, and A. Cox, "Homeostatic utility control," IEEE Trans. Power App. Syst., vol. 99, no. 3, pp. $1151-1163,1980$.

[10] H. Glatvitsch and F. Alvarado, "Management of multiple congested conditions in unbundled operation of a power system," IEEE Trans. Power Systems, vol. 13, no. 3, pp. 1013 - 1019, 1998.

[11] F. Alvarado, "Controlling power systems with price signals," Decis. Support Syst., vol. 40, no. 3 - 4, pp. 495 - 504, 2005.

[12] A. Joki, M. Lazar, and P. van den Bosch, "Real-time control of power systems using nodal prices," Int. J. Elec. Power Energy Syst., vol. 31, no. 9 , pp. $522-530,2009$.

[13] D. Hammerstrom, "Pacific Northwest GridWiseTM testbed demonstration projects. Part I. Olympic Peninsula project," Tech. Rep. PNNL17167, Pacific Northwest National Laboratory (USA), 2007.

[14] Actua, Enfor, Eurisco, E. Power, S.Nve, E. E. Analyses, and DTU, "FlexPower," E.A. Energy Analyses (Denmark), Tech. Rep., 2010, available at: http://ea-energianalyse.dk.

[15] H. Allcott, "Rethinking real time electricity pricing," Tech. Rep. 20090015, MIT Center for Energy and Environmental Policy Research, 2009.

[16] K. Heussen, S. You, B. Biegel, L. Hansen, and K. B. Andersen, "Indirect control for demand side management - A conceptual introduction," in Proc. of the 3rd IEEE PES Innovative Smart Grid Technologies (ISGT) Europe Conference, 2012.

[17] M. Roozbehani, M. Dahleh, and S. Mitter, "Volatility of power grids under real-time pricing," IEEE Trans. Power Syst., 2012, available online.

[18] M. Zugno, J. Morales, P. Pinson, and H. Madsen, "A bilevel model for electricity retailers participation in a demand response market environment," Energ. Econ., vol. 36, pp. 182 - 197, 2013.

[19] O. Corradi, H. Ochsenfeld, H. Madsen, and P. Pinson, "Controlling electricity consumption by forecasting its response to varying prices," IEEE Trans. Power Syst., vol. 28, no. 1, pp. 421 - 429, 2013.

[20] P. Richardson, A. Keane, and D. Flynn, "Optimal charging of electric vehicles in low-voltage distribution systems," IEEE Trans. Power Syst., vol. 27, no. 1, pp. $268-279,2012$.

[21] A. Roscoe and G. Ault, "Supporting high penetrations of renewable generation via implementation of real-time electricity pricing and demand response," Renew. Power Gen., vol. 4, no. 4, pp. 369 - 382, 2010.

[22] M. D. Ilic, L. Xie, and J.-Y. Joo, "Efficient coordination of wind power and price-responsive demand part i: Theoretical foundations," IEEE Trans. Power Syst., vol. 26, no. 4, pp. 1875-1884, 2011.

[23] H. Madsen, Time Series Analysis. Chapman \& Hall/CRC Texts in Statistical Science, 2008.

[24] L. Andrieu, R. Henrion, and W. Römisch, "A model for dynamic chance constraints in hydro power reservoir management," Eur. J. Oper. Res., vol. 207, no. 2, pp. 579 - 589, 2010.

[25] J. Bear and Y. Sun, "Optimization of pump-treat-inject (PTI) design for the remediation of a contaminated aquifer: Multi-stage design with chance constraints," J. Contam. Hydrol., vol. 29, no. 3, pp. 225 - 244, 1998.

[26] N. H. Agnew, R. A. Agnew, J. Rasmussen, and K. R. Smith, “An application of change constrained programming to portfolio selection in a casualty insurance firm." Manage. Sci., vol. 15, no. 10, pp. 512 $520,1969$.
[27] R. Henrion and A. Moller, "Optimization of a continuous distillation process under random inflow rate," Comput. Math. Appl., vol. 45, no. 1 - 3, pp. 247 - 262, January 2003.

[28] Q. Wang, Y. Guan, and J. Wang, "A Chance-Constrained Two-Stage Stochastic Program for Unit Commitment With Uncertain Wind Power Output," Power Systems, IEEE Transactions on, vol. 27, no. 1, pp. 206 $-215,2012$.

[29] H. Zhang and P. Li, "Chance Constrained Programming for Optimal Power Flow Under Uncertainty," Power Systems, IEEE Transactions on, vol. 26, no. 4, pp. 2417 - 2424, 2011.

[30] U. A. Ozturk, M. Mazumdar, and B. A. Norman, "A solution to the stochastic unit commitment problem using chance constrained programming," Power Systems, IEEE Transactions on, vol. 19, no. 3, pp. 1589 - 1598, 2004.

[31] A. Shapiro, D. Dentcheva, and A. Ruszczynski, Lectures on Stochastic Programming: Modeling and Theory. SIAM, Philadelphia, 2009, vol. 9.

[32] A. Prekopa, Stochastic Programming. Kluwer Academic Publishers, Dordrecht, The Netherlands, 1995.

[33] A. Ben-Tal and A. Nemirovski, Lectures on Modern Convex Optimization. Analysis, Algorithms, and Engineering Applications. SIAM, Philadelphia, 2001.

[34] S. Boyd and L. Vandenberghe, Convex Optimization. Cambridge University Press, 2004. [Online]. Available: www.stanford.edu/ boyd/cvxbook.html

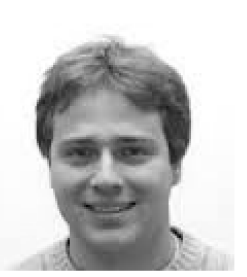

Gianluca F Dorini received the M.Sc. in Environmental Engineering at the Politecnico di Milano (Milan, Italy), and the PhD degree in Water Systems Engineering at the University of Exeter (United Kingdom). He is Post-doctoral fellow at the Department of Applied Mathematics and Computer Sceince the Technical University of Denmark. His main research interests are modelling and optimization, applied to planning and management of natural resources. His experience covers asset management, ground fow modeling and optimal management, sustainable planning in urban environment, security in water distribution networks, multiobjective Markov Decision Process, and their application to multi objective reservoir operation.

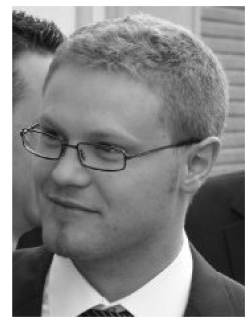

Pierre Pinson (M'11, SM'13) received the M.Sc. degree in Applied Mathematics from the National Institute for Applied Sciences (INSA Toulouse, France) and the Ph.D. degree in Energy from Ecole des Mines de Paris. He is the Professor in Modelling of Electricity Markets at the Technical University of Denmark, Centre for electric Power and Energy, Dpt. of Electrical Engineering. His research interests include among others forecasting, uncertainty estimation, optimization under uncertainty, decision sciences, and renewable energies. He acts as an Editor for the IEEE Transactions on Power Systems and for Wind Energy.

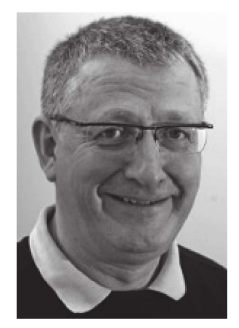

Henrik Madsen received a Ph.D. in Statistics at the Technical University of Denmark in 1986. He was appointed Professor in Mathematical Statistics in 1999. He is an elected member of the International Statistical Institute (ISI), and he has participated in the development of several ISO and CEN standards. His research interests includes analysis and modeling of stochastic dynamics systems, signal processing, time series analysis, identification, estimation, grey-box modeling, prediction, optimization and control, with applications mostly related to energy systems, informatics, environmental systems, bioinformatics, biostatistics, process modeling and finance. 